\title{
Annexe 1 - Rapport du Conseil fédéral sur les relations Nord-Sud de la Suisse dans les années 90 (Lignes directrices Nord-Sud)
}

\section{(2) OpenEdition}

\section{Journals}

Édition électronique

URL : http://journals.openedition.org/aspd/1486

DOI : 10.4000/aspd. 1486

ISSN : 1663-9669

Éditeur

Institut de hautes études internationales et du développement

Édition imprimée

Date de publication : 1 février 1995

Pagination : 218-232

ISSN : 1660-5934

Référence électronique

"Annexe 1 - Rapport du Conseil fédéral sur les relations Nord-Sud de la Suisse dans les années 90 (Lignes directrices Nord-Sud)». Annuaire suisse de politique de développement [En ligne], 14 | 1995, mis en ligne le 04 mai 2013, consulté le 08 septembre 2020. URL : http://journals.openedition.org/aspd/ 1486 ; DOI : https://doi.org/10.4000/aspd.1486 
Annexe 1

\section{Rapport du Conseil fédéral sur les relations Nord-Sud de la Suisse dans les années 90 (Lignes directrices Nord-Sud)}

\section{Résumé}

Les Lignes directrices Nord-Sud, font suite à un postulat de la Commission des affaires étrangères du Conseil des États du 6 juin 1990.

Ce postulat demandait au Conseil fédéral d'élaborer, dans le cadre d'une analyse supra-départementale et d 'une discussion de principes, un plan directeur du rôle futur de la Suisse dans les relations Nord-Sud, en vue d'une politique de développement globale et cohérente.

Les lignes directrices ont été élaborées parallèlement au Rapport sur la politique extérieure de la Suisse. Le Conseil fédéral a mentionné dans ce rapport qu'il présentera un rapport séparé consacré aux lignes directrices des relations de la Suisse avec les pays en développement et qui approfondirait le thème de la cohérence».

Les lignes directrices exposent les problèmes principaux d'une politique de développement à l'heure actuelle Elles en montrent les conséquences et les champs d'action et établissent les principes directeurs que suivra à l'avenir une politique suisse de développement $\mathrm{Ce}$ n'est plus seulement la coopération au développement qui est concernée, mais bien l'ensemble des relations de la Suisse avec les pays en développement.

Les lignes directrices visent les mêmes objectifs que le rapport sur la politique extérieure et reposent sur la sauvegarde à long terme des intérêts suisses.

\section{Rapport}

1. Contexte d'une politique de développement dans les années 90

1.1. Globalisation dans les domaines de la politique, de l'environnement et de l'économie

Dès le début des années 90 , les relations traditionnelles entre les pays industrialisés et les pays en développement, entre le Nord et le Sud se sont fondamentalement transformées. La fin de la guerre froide et la Conférence sur l'environnement et le développement à Rio en 1992 caractérisent une période de profonds changements au plan global ainsi qu'une nouvelle perception du monde. 
Le conflit idéologique est-ouest qui dominait jusqu'en 1989 a fait place, dans les pays industrialisés comme dans les pays en développement, à une nouvelle orientation dont les contours sont déjà visibles, une globalisation de valeurs fondamentales: le principe d'une société ouverte et démocratique, le rôle du marché en tant que principe d'ordre économique et le respect nécessaire de l'équilibre écologique. Les relations entre les pays industrialisés et les pays en développement ont ainsi changé, la politique de force qui divisait le Sud en deux sphères d'influence a disparu. Mais l'euphorie de 1989 et l'espoir d'un nouvel ordre mondial se sont envolés, alors que des nationalismes anciens et des conflits ethniques refont surface avec plus ou moins de violence, non seulement dans des pays en développement lointains, mais aussi en Europe, dans l'ex-Yougoslavie, au Proche-Orient et dans une partie du bassin méditerranéen. Cet amalgame imprévisible de chances et de risques place les gouvernements, ainsi que les organisations internationales, devant des tâches nouvelles.

La Conférence de Rio a montré à un vaste public la globalisation des problèmes de notre environnement naturel. Les pays en développement y jouent un rôle important. Ils détiennent une grande partie des ressources naturelles et sont bien plus menacés que les pays industrialisés par les conséquences de la désertification, l'élévation du niveau de la mer ou les changements climatiques. La pauvreté, l'industrialisation et la croissance démographique portent une atteinte supplémentaire à l'environnement. En outre, il est admis qu'une généralisation du mode de vie occidental actuel n'est en principe plus concevable écologiquement.

Les moyens modernes de communication et de transport, la mobilité croissante des marchandises, des services, des capitaux et de la main-d'œuvre ainsi que l'accélération de la division internationale du travail encouragent et exigent une globalisation de l'économie. Face au développement dynamique de l'économie privée, les travaux des organisations multilatérales qui visent à établir le cadre juridique institutionnel de ce processus sont plus ardus. Dans de nombreux pays industrialisés, les craintes face à la progression du chômage alimentent des courants protectionnistes. Si la conclusion positive du Cycle d'Uruguay ne parvient pas à freiner cette tendance, de nombreux pays en développement seront touchés à un moment où, à des coûts sociaux souvent élevés, ils ont amélioré les conditions de leur économie d'exportation. En outre, les pays d'Europe centrale et orientale, ainsi que ceux de l'ancienne Union soviétique, leur font concurrence sur les marchés des pays de l'OCDE.

\subsection{La fin du "tiers monde"}

Les pays en développement se trouvent actuellement à des stades très différents de développement. Des pays comme la Corée du Sud, le Chili ou le Mexique présentent des caractéristiques de pays industrialisés, alors que les pays les plus pauvres tels le Népal, le Mali ou Haïti affrontent encore des problèmes politiques, économiques et écologiques vitaux. 
Entre ces deux extrêmes, toutes les formes existent. Dans de grands pays, tels que l'Inde, le Brésil, I'Indonésie ou la Chine, les réalités d'un pays industrialisé très développé se mêlent aux indicateurs de pauvreté d'un pays en développement. Ces disparités entre les pays et les régions, souvent au sein d'un même État ou d'une même société, risquent de s'aggraver dans les années à venir. Ces tendances font apparaître un développement inégal, voire un monde à deux vitesses composé de gagnants et de perdants. La notion de utiers monde", terme générique qui désignait l'ensemble des pays en développement, n'en englobe plus la totalité.

Le bilan des efforts de développement des trois dernières décennies est contrasté. Certains pays en développement ont su profiter de ces dernières années pour améliorer leur mode de gouvernement et mettre en œuvre une politique économique plus efficace. Dans les domaines de la santé et de l'éducation, les disparités entre le Nord et le Sud se sont nettement atténuées, bien que la pandémie du Sida remette en cause les progrès réalisés dans plusieurs régions pauvres d'Afrique et d'Amérique latine. La production alimentaire mondiale a triplé pendant cette période. Jamais auparavant les conditions de vie d'un aussi grand nombre d'individus n'avaient été améliorées dans un délai aussi court.

Cependant, plus d'un milliard de personnes vivent dans la pauvreté absolue, ce qui signifie qu'elles ne disposent pas d'un revenu suffisant pour satisfaire leurs besoins fondamentaux: alimentation, eau propre, vêtements et logement. Beaucoup de pays en développement ne sont pas encore gouvernés par des autorités responsables, orientées vers le développement, soucieuses du bien-être de la population, capables d'engager des ressources limitées d'une manière efficace, d'élaborer des conditions cadres économiques et politiques claires et de définir des priorités. En Afrique subsaharienne (sans l'Afrique du Sud), le développement stagne. D'ici à l'an 2020, la population mondiale passera probablement de 5,4 à 8 milliards d'individus. L'exode rural et les mouvements migratoires transfrontaliers et transcontinentaux compromettront en maint endroit la paix sociale.

\subsection{La nouvelle dépendance entre le Nord et le Sud}

La croissance démographique, la pauvreté, l'endettement et la stagnation économique se traduisent par un chômage croissant, par la criminalité, par la destruction de la nature et par l'exode rural. Ces problèmes se renforcent réciproquement, exercent leur influence au-delà des frontières et façonnent l'avenir.

Pauvreté et industrialisation peuvent devenir des problèmes environnementaux globaux qui auront des répercussions sur les générations futures. La destruction des forêts tropicales nous en donne déjà la preuve. La pauvreté, la croissance démographique et l'exode rural au Sud renforcent les migrations et compromettent la paix sociale au Nord. Inversement, par leurs politiques des taux d'intérêt, des taux de change, des mouvements de capitaux, de l'immigra- 
tion, de l'emploi et de l'industrialisation, ou encore par leurs politiques du commerce extérieur et de l'environnement, les pays industrialisés ont une influence directe sur l'avenir des pays en développement.

\subsection{Nécessité d'une politique cohérente à l'égard du Sud}

La Suisse est ainsi confrontée au défi de repenser ses relations avec les pays en développement, dans un contexte dynamique et complexe. Ce n'est plus seulement la coopération au développement qui est mise en cause mais l'ensemble de nos relations politiques, économiques et sociales avec les pays du Sud. Les dichotomies traditionnelles entre politique de l'environnement et politique économique, entre politique économique et politique des migrations, entre politique commerciale et politique de coopération au développement, entre politique intérieure et politique extérieure, ne permettent plus de répondre aux problèmes actuels. Ce qu'il faut, c'est "une politique cohérente envers le Sud". Formuler une telle politique revient à montrer les contradictions éventuelles entre des intérêts nationaux à court terme et les buts de la politique suisse de développement, puis à les intégrer, de façon aussi transparente que possible, dans les processus de décisions politiques.

Une telle politique ne peut être mise en œuvre que si la population suisse réalise que notre prospérité dépend à long terme du destin du Sud. Les présentes lignes directrices Nord-Sud doivent contribuer à une meilleure compréhension de ces interrelations.

\subsection{Les lignes directrices Nord-Sud et la politique extérieure suisse}

Dans son "Rapport sur la politique extérieure de la Suisse dans les années 90" présenté le 29 novembre 1993, le Conseil fédéral fixe les objectifs stratégiques de la politique extérieure globale:

- le maintien et la promotion de la sécurité et de la paix,

- l'engagement en faveur des droits de l'homme, de la démocratie et des principes de l'État de droit,

- l'accroissement de la prospérité commune,

- la promotion de la cohésion sociale,

- la préservation du milieu naturel.

Ces objectifs sont valables pour l'ensemble de notre politique extérieure, dans nos relations avec nos voisins européens aussi bien qu'avec les pays en développement. Les lignes directrices Nord-Sud ont été élaborées en étroite concordance avec le Rapport sur la politique extérieure. Elles visent les mêmes objectifs et reposent sur la sauvegarde à long terme des intérêts suisses.

La mise en œuvre des objectifs développés ci-après ne peut se concevoir ni se réaliser sans le soutien de la population. Il est nécessaire de trouver un bon équilibre entre intérêts à court terme et intérêts à long terme, entre intérêts nationaux et intérêts internationaux. II n'est pas question d'abandonner les 
intérêts suisses. Cependant, dans un monde que caractérisent l'interdépendance et la mutation, nous devons en permanence, dans une perspective à long terme et globalement, redéfinir les intérêts afin de mieux les préserver et déterminer les actions qui en découlent afin de mieux les conduire.

\section{Lignes directrices d'une politique de développement pour les années 90}

2.1. Sauvegarder et maintenir la paix et la sécurité, promouvoir les droits de l'homme, la démocratie et l'État de droit

Les droits de l'homme, la démocratie, de droit et la liberté dans la paix sont des valeurs fondamentales, essentielles pour le fonctionnement de l'appareil étatique suisse et dont nous affirmons la signification universelle. Ces principes sont aussi indispensables à la stabilité politique dans les pays en développement. Ils restreignent les probabilités de conflits intérieurs et extérieurs. Ils permettent à l'État, à la société, aux bailleurs de fonds étrangers d'utiliser le temps, l'énergie et les moyens financiers à disposition pour faire réellement face aux défis économiques, sociaux et écologiques. Ils renforcent la prospérité et freinent les migrations. L'amélioration des conditions cadres politiques dans les pays en développement est ainsi dans l'intérêt à long terme de notre propre avenir.

\subsubsection{Promouvoir la bonne gestion des affaires publiques}

Les instances fédérales compétentes appliquent les principes "de bonne gestion des affaires publiques" dégagés par l'OCDE et à l'élaboration desquels la Suisse a activement collaboré, pour promouvoir le respect des droits de l'homme, la démocratie et l'État de droit dans les pays du Sud. Des mesures positives (soutien à des organisations locales de défense des droits de I'homme, appui aux processus électoraux, programmes de formation pour l'administration et l'appareil judiciaire, promotion des structures administratives de l'État de droit, etc.), le dialogue politique avec les pays partenaires sur les conditions cadres requises pour une coopération efficace et, dans certains cas, la conditionnalité directe qui lie l'octroi de l'aide à certaines conditions politiques et économiques permettent cette promotion.

\subsubsection{Promouvoir les droits de l'homme, de droit et le processus de dé- mocratisation par des mesures positives}

Les programmes et les projets de la coopération suisse au développement sont examinés quant à leur conformité avec les critères de respect des droits de l'homme et quant à leur influence sur la promotion de l'État de droit. De plus, des mesures positives en vue de renforcer le respect des droits de l'homme, de droit et les processus démocratiques sont de plus en plus souvent intégrées à la coopération au développement. 


\subsubsection{Montrer les conflits d'objectifs}

Entre la politique intérieure en faveur de l'emploi et de la croissance économique, grâce à des mesures étatiques de promotion des exportations, et la politique de développement en faveur de la démocratie et des droits de l'homme, des contradictions peuvent surgir qu'il faut alors mettre en évidence et clarifier par le dialogue. Dans l'appréciation des risques en vue de l'octroi de la garantie aux risques à l'exportation (GRE) pour des livraisons dans les pays en développement les plus pauvres, il est nécessaire de mettre plus d'accent sur les aspects d'ordre politique et sur le respect des droits de l'homme dans les pays récipiendaires. II convient, si possible, de rechercher une action internationale concertée.

\subsubsection{Aménager l'assistance judiciaire internationale}

Les mesures visant à interdire le transfert et le rapatriement de capitaux acquis illégalement à l'étranger ou transférés illégalement à l'étranger sont renforcées. A cet effet, le Conseil fédéral révise actuellement la loi sur l'assistance judiciaire afin d'accélérer les procédures judiciaires. Sur le plan international, la Suisse s'engage en faveur d'une harmonisation des dispositions légales pour empêcher que les capitaux en fuite, originaires de pays en développement, ne puissent profiter de dispositions légales plus favorables sur d'autres places financières. Le Conseil fédéral étudie en outre d'autres mesures concrètes permettant, dans le cadre de la coordination internationale, de combattre la corruption.

\subsubsection{Réduire les dépenses militaires exagérées des pays en développe- ment}

La Suisse appuie des mesures positives visant à renforcer la sécurité et la paix et à réduire les dépenses militaires des pays partenaires ( $p$. ex. programmes de démobilisation et de réintégration civile). Dans le cadre du dialogue politique avec le pays bénéficiaire, aux niveaux bilatéral et multilatéral, en particulier dans les instances de coordination internationale de la Banque mondiale et du PNUD (groupes consultatifs, tables rondes), la Suisse s'engage fermement en faveur d'une réduction des dépenses militaires excessives afin que la plus grande part possible des ressources financières limitées soit allouée au développement économique et social.

Dans le cadre de la révision actuelle de la loi sur le matériel de guerre, il convient de prendre en considération qu'une autorisation d'exporter des armes n'est accordée que si elle n'entre pas en conflit avec les principes fondamentaux de la politique extérieure suisse. Toute décision dépend notamment de savoir si l'exportation ne compromet ni le maintien de la paix, ni le respect des droits de l'homme, et si l'autorisation n'entre pas en conflit avec les principes de la politique suisse de développement et avec les efforts engagés dans le do- 
maine de la coopération au développement. La Suisse s'engage sur le plan multilatéral en faveur de l'harmonisation des législations nationales qui limitent les exportations de matériel de guerre excessives et freinent le développement.

\subsubsection{Rétablir et sauvegarder la paix}

La Suisse renforce sa contribution aux efforts internationaux pour la sauvegarde et le maintien de la paix et de la sécurité par une participation accrue à des actions de maintien de la paix. A cet effet, elle met notamment sur pied un contingent suisse de casques bleus. Elle prévoit d'augmenter ses ressources financières et humaines dans le domaine de la diplomatie préventive, de la solution des crises, du contrôle des armements et du désarmement. La Suisse s'engage de façon accrue au sein de la CSCE et de l'ONU dans le domaine de la prévention des conflits et de la solution des crises. Afin d'être en mesure d'intervenir de plein droit dans les institutions et les commissions des Nations Unies importantes pour la paix et la sécurité, le Conseil fédéral tient toujours à ce que la Suisse devienne membre à part entière de l'ONU. La Suisse soutient, au travers de mesures stimulant la confiance et la sécurité, les organisations régionales de sécurité collective hors d'Europe.

\subsection{Promouvoir la prospérité}

La politique économique de nombreux pays en développement se caractérisait dans le passé par une monnaie surévaluée, une inflation élevée, des déficits budgétaires, un endettement extérieur, des subventions à des entreprises étatiques non rentables, des marchés protégés, et par une dépendance due à des échanges désavantageux ainsi qu'à des prix et des taux d'intérêt soumis aux fluctuations internationales. Cette situation a obligé presque tous les gouvernements à mettre en œuvre les ajustements structurels nécessaires.

Seule une répartition nouvelle des tâches entre l'État et la société civile, respectivement l'économie privée, peut assurer le succès durable des réformes entreprises. II appartient à l'État de mettre en place des conditions cadres qui encouragent le développement des initiatives privées et qui assurent une croissance durable, socialement équilibrée. L'utilisation efficace des fonds de la coopération internationale y concourt.

Seul un développement économique durable permet la création de postes de travail et donc de revenus, donne aux populations du Sud des perspectives d'avenir et offre d'autres solutions que l'émigration. Le développement durable est une condition nécessaire à la réduction de la pauvreté, à la diminution de la croissance démographique et à la protection de l'environnement.

\subsubsection{Améliorer les conditions cadres pour un développement durable des pays en développement}

Avant d'atteindre une croissance économique durable, les pays les plus pau- 
vres dépendront longtemps de l'aide extérieure. Les ressources humaines et financières leur manquent. Les pays à revenu moyen ont également besoin de notre soutien pour améliorer leurs infrastructures économiques et sociales et pour protéger efficacement leur environnement. Notre aide publique au développement doit donc encore augmenter quantitativement et qualitativement. Pour sauvegarder à long terme les intérêts propres de la Suisse et pour assumer sa part de responsabilités au sein de la communauté internationale, le Conseil fédéral maintient l'objectif de porter, dans toute la mesure du possible et jusqu'au tournant du millénaire, le volume de l'aide publique à $0,40 \%$ du produit national brut.

La coopération suisse au développement a pour première priorité l'amélioration des ressources productives, en favorisant la production agricole et la sécurité alimentaire, en promouvant la production industrielle et artisanale, en soutenant le développement de technologies adaptées. II s'agit de soutenir financièrement des ajustements structurels socialement supportables. Cet appui prend aussi les formes suivantes: soutien aux capacités de recherche scientifique, en particulier dans l'agriculture, soutien aux infrastructures de transport, promotion de la diversification économique (secteur informel, petites entreprises, nouveaux domaines d'activités axées sur l'exportation) et promotion du secteur privé au sens le plus large.

Nous sommes toujours à la recherche de moyens et de méthodes permettant d'accroître l'efficacité et la pertinence de l'aide au développement. Nous insistons sur une coordination accrue avec les autres bailleurs de fonds et sur le développement de synergies entre les différents instruments de notre coopération. Les efforts de développement ne peuvent être durables que si nos partenaires ont la maîtrise de leur développement ("empowerment"). Nos partenaires sont encouragés à poursuivre des buts à long terme pour lesquels les bénéficiaires eux-mêmes doivent s'engager financièrement.

La politique internationale des produits de base est dans l'impasse: les accords de stabilisation n'ont pu empêcher de larges fluctuations de prix et une détérioration continue des termes de l'échange pour les pays en développement. La Suisse participera activement aux travaux de la CNUCED et de la Banque mondiale pour formuler une politique nouvelle. Le programme suisse de compensation des pertes de recettes à l'exportation des pays en développement ("STABEX suisse") sera évalué, notamment quant à son efficacité à favoriser des changements structurels dans les pays en développement.

Le fardeau trop lourd du service de la dette reste un obstacle fondamental au développement d'un grand nombre de pays parmi les plus pauvres. Dans le cadre de son programme de désendettement, la Suisse met en cuuvre des mesures de désendettement bilatérales et soutient des efforts multilatéraux. Au sein du "Club de Paris" (qui regroupe les principaux pays créanciers), elle soutient des scénarios de désendettement réalistes en faveur des pays les plus pauvres lourdement endettés.

La corruption sévit dans nombre de pays. Les pays donateurs et les institutions multilatérales font des efforts considérables pour contrôler l'utilisation des 
fonds qu'ils engagent. La Suisse appuie les travaux entrepris dans le cadre de I'OCDE pour élaborer un code de conduite visant à lutter contre la corruption dans les pays industrialisés.

\subsubsection{Promouvoir le secteur privé dans les pays en développement}

Le retrait de l'État des secteurs productifs entraîne une responsabilité très grande de l'économie privée dans l'instauration d'un développement durable. La Suisse cherche à créer un cadre favorable aux initiatives de petites et moyennes entreprises, d'artisans et de paysans. Elle encourage la mise en place et le développement d'organisations au sein de la société civile ( $p$. ex. organisations paysannes, associations professionnelles). Elle s'engage dans la formation professionnelle et elle soutient les très petites, petites et moyennes entreprises. Ces mesures devraient permettre aux populations concernées d'envisager un futur viable dans leur propre pays. Elles constituent également une réelle solution de rechange aux migrations non réglementées. II faut cependant rester attentif au conflit d'intérêt potentiel entre, d'une part, les mesures préventives et les moyens mis en œuvre dans les pays d'origine des migrants du point de vue de la politique de migration et, d'autre part, les objectifs de la coopération au développement. Afin de relativiser ce conflit, il faut partir du principe que les mouvements migratoires non réglementés ne constituent une solution ni pour les pays en développement, ni pour les pays industrialisés.

La coopération suisse au développement est en train de mettre au point un instrument qui permette, sans augmenter la dette, de stimuler l'investissement privé dans le Sud, en particulier dans les pays à revenu moyen. Cet instrument a pour objectif essentiel d'encourager un partenariat entre le secteur privé suisse et le secteur privé des pays en développement. Ce nouvel instrument doit englober les instruments traditionnels de coopération économique (crédits mixtes, promotion commerciale et de l'investissement, transfert de technologie), permettre une approche globale dans l'analyse des besoins, et combiner assistance technique et aide financière.

\subsubsection{Faciliter l'accès aux marchés du Nord pour les produits des pays en développement}

L'établissement d'un système commercial multilatéral ouvert est essentiel pour ces pays. Seules des recettes d'exportation croissantes leur permettront de financer, par leurs propres ressources, leurs besoins dans une proportion accrue, de créer des places de travail et d'offrir un avenir à une population en augmentation. II est de notre intérêt d'améliorer l'accès aux marchés des produits des pays en développement. Même si une telle ouverture peut avoir à court terme des effets sur le marché du travail en Suisse, le chômage ne trouvera pas de solution à long terme dans une politique protectionniste.

Le Cycle d'Uruguay aura des effets positifs pour nombre de pays en développement. Les recettes d'exportation qui en découleront sont estimées annuel- 
lement à quelque 65 milliards de dollars américains, ce qui dépasse le montant total de l'aide au développement des pays de l'OCDE en 1992 (60 mia US \$). La baisse des droits de douane, la suppression progressive des restrictions à l'importation dans le domaine des textiles, la suppression de quotas et d'autres restrictions quantitatives dans le secteur agricole jouent en faveur des pays en développement. Toutefois, la libéralisation ne peut que créer des opportunités dont seuls les pays et les entreprises les plus compétitifs pourront profiter. Les pays qui ne le pourront auront besoin d'une aide accrue qui leur permettra d'augmenter leur compétitivité et de se dégager progressivement de leur situation de bénéficiaires de l'aide.

\subsection{Améliorer la justice sociale}

La justice sociale, la faim et la pauvreté figurent encore au nombre des problèmes les plus importants de notre époque. Ils exigent notre solidarité non seulement par principe humanitaire mais parce qu'ils engendrent la croissance démographique, la destruction de l'environnement et les flux migratoires. Ils provoquerit des tensions qui menacent la paix et la sécurité. La Suisse a donc un intérêt vital à améliorer les conditions sociales cadres dans les pays en développement.

\subsubsection{Lutter contre la pauvreté et promouvoir la justice sociale}

La coopération au développement représente l'instrument de politique étrangère le plus important dans la lutte contre la pauvreté et la promotion de la justice sociale à l'échelle mondiale. II reste nécessaire d'en accroître l'efficacité et d'en améliorer la coordination au plan international. Les politiques d'immigration, d'asile et de coopération au développement doivent être cohérentes afin que les conditions économiques et politiques soient telles dans le plus grand nombre de pays et de régions qu'elles permettent aux populations d'y vivre décemment.

Par le dialogue politique avec les pays récipiendaires, la Suisse désire encourager une politique sociale et économique orientée vers la lutte contre la pauvreté, une politique qui fait encore défaut dans nombre de pays en développement. Elle soutient leur politique sociale par des mesures appropriées dans les domaines de l'éducation, de la santé, de la démographie, et notamment aussi dans la lutte contre la propagation du Sida.

Un des objectifs définis par la Conférence des Nations Unies pour les pays les moins avancés (PMA) doit être atteint au cours des années 90: l'aide au développement en faveur des pays les plus pauvres qui ont décidé de donner la priorité à la lutte contre la pauvreté devrait représenter au moins 0,15 pour cent du produit national brut. De même, la part actuelle de l'aide publique au développement allouée au domaine social doit être accrue de manière significative d'ici à l'an 2000.

Dans le cadre de sa participation aux Institutions de Bretton Woods, aux 
banques et aux fonds régionaux de développement et aux organisations des Nations Unies, la Suisse œuvre pour que ces institutions centrent leurs activités sur la lutte contre la pauvreté. Elle s'engage aussi pour une prise en compte plus déterminée des coûts sociaux et écologiques de l'ajustement structurel.

\subsubsection{Freiner la croissance démographique}

La contribution suisse à la politique démographique doit être renforcée, en premier lieu dans le cadre de programmes multilatéraux et en second lieu dans le cadre bilatéral. Les mesures relatives à la croissance de la population et au contrôle des naissances doivent respecter l'autonomie et les valeurs culturelles des pays en développement concernés; elles doivent être intégrées aux programmes mis en œuvre dans le secteur de la santé et de l'éducation. L'amélioration du statut de la femme et l'accès de cette dernière à l'emploi, à l'éducation et aux soins de santé revêtent une grande importance.

\subsubsection{Promouvoir une aide humanitaire cohérente et coordonnée à l'échelle internationale}

Le nombre croissant de foyers de conflits dans maintes régions implique une aide humanitaire accrue et rapide. Une coordination internationale renforcée doit rendre l'aide humanitaire encore plus efficace. II convient de procéder avec soin, en collaboration avec des organisations suisses et internationales, à une clarification des champs de compétences et de viser une coordination efficace, sur le terrain, des apports d'aide humanitaire. Un dialogue politique plus intense avec les organisations internationales avec lesquelles nous collaborons joue à cet égard un rôle déterminant.

L'aide humanitaire ne doit pas conduire à de nouveaux rapports de dépendance. Dans toute la mesure du possible, elle doit être une aide à l'autopromotion et doit déboucher le plus rapidement possible sur une aide à la reconstruction. Une harmonisation est indispensable avec d'autres instruments de politique extérieure. L'aide alimentaire doit avoir pour objectif général la sécurité alimentaire. Si cela s'avère économiquement et logistiquement possible, cette aide doit contribuer au soutien de la production agricole par l'achat de produits dans la région concernée.

Dans les pays en crise ou en guerre, la sécurité doit être garantie avant tout au niveau régional. Les déplacements de populations vers les pays industrialisés ne doivent être envisagés que comme solutions de dernier recours. La Suisse oeuvre dans ce contexte pour un partage équilibré des responsabilités au niveau international.

\subsection{Protéger l'environnement naturel}

La Conférence des Nations Unies sur l'environnement et le développement (CNUED), qui s'est tenue à Rio en juin 1992, a montré de manière convain- 
cante que le passage à un modèle de développement durable et respectueux de l'environnement est devenu, à l'échelle mondiale, une question de survie pour notre terre. Au Nord comme au Sud, des adaptations importantes sont nécessaires pour que l'on s'achemine vers un développement durable; seule une coopération étroite entre pays industrialisés et pays en développement permettra d'y parvenir. L'un des défis majeurs à l'avenir de la politique de développement est de faire accepter socialement et politiquement, au Nord comme au Sud, les changements nécessaires de notre façon de vivre et de l'utilisation que nous faisons de ressources naturelles limitées.

\subsection{1. Élaborer un modèle de développement durable en Suisse}

Le mode de consommation des pays industrialisés et leur utilisation immodérée de ressources sont responsables, pour une part plus que proportionnelle, des effets négatifs sur l'environnement et ne doivent pas servir de modèles aux pays en développement. C'est la raison pour laquelle la Suisse doit contribuer, à l'intérieur de ses frontières aussi, à un développement durable. L'une des étapes nécessaires est la ratification et la mise en œuvre d'accords internationaux, notamment des conventions sur le climat et sur la biodiversité. De plus, un plan d'action national et multisectoriel, donnant suite à l'Agenda 21 de la CNUED, est élaboré et mis en œuvre pour permettre à notre pays de passer à un modèle de développement durable.

La Suisse développe des stratégies de croissance qualitative, afin de maintenir la qualité de vie tout en diminuant le gaspillage de ressources. Elle prépare des mesures d'application basées sur le principe du pollueur-payeur et sur l'internalisation des coûts extérieurs supportés jusqu'à présent par la communauté.

La Suisse encourage une utilisation rationnelle des ressources naturelles, y compris des agents énergétiques, le recyclage et la régénération des ressources employées. Dans le cadre du Programme "Énergie 2000", elle poursuit la stabilisation au niveau de 1990 de la consommation d'énergie fossile et des émissions de CO2 d'ici à l'an 2000, puis elle réduira les émissions de CO2 et d'autres gaz à effet de serre qui ne sont pas encore réglementés par le Protocole de Montréal.

La Suisse promeut la mise en œuvre d'un modèle de développement durable par le secteur privé, notamment en déterminant les conditions cadres.

\subsubsection{Faire comprendre l'importance d'un développement durable sur le plan international}

La Suisse participe activement au processus de suivi de la Conference de Rio (CNUED). Elle œuvre notamment pour que la Commission sur le développement durable, mise sur pied par les Nations Unies, devienne un organe efficace de coordination, de contrôle et d'action. Au sein des institutions financières internationales de développement (Banque mondiale, banques régionales, or- 
ganisations des Nations Unies), les représentants suisses défendent une ligne d'action conforme aux exigences d'un développement durable. Ils demandent en particulier que les décisions de financement soient précédées d'une analyse appropriée de l'impact sur l'environnement.

Les programmes de la coopération bilatérale suisse doivent soutenir les efforts que déploient les pays les plus démunis en vue de parvenir à un développement durable. A cet effet, le volume de l'aide publique au développement doit être augmenté et les accents doivent être mis en fonction des exigences d'un développement durable. Une attention toute particulière doit être portée au développement des ressources humaines (éducation et santé) et au renforcement de la capacité de gestion des pays en développement afin qu'ils parviennent à une maîtrise durable de l'utilisation de leurs ressources naturelles. Les secteurs prioritaires sont le développement des régions de montagne, la protection et l'exploitation raisonnée des forêts tropicales, la conservation des sols, l'eau potable et la réduction de déchets.

La coopération en matière de recherche scientifique sur des thèmes relatifs à l'environnement global est soutenue par la mise en place d'un partenariat de recherche entre les hautes écoles suisses et les institutions de recherche du Sud. La Suisse soutient également la coopération technologique en matière d'environnement entre l'économie privée des pays industrialisés et celle des pays en développement.

\subsubsection{Harmoniser les politiques de l'environnement, du commerce et du développement}

Les contradictions qui existent entre les politiques de l'environnement, du commerce et du développement doivent être surmontées dans toute la mesure du possible. Dans les négociations multilatérales, la Suisse œuvre pour que les objectifs économiques, environnementaux et de développement soient intégrés, sous une forme adéquate, aux principaux accords multilatéraux dans le domaine du commerce, des communications, des transports et de l'énergie.

Les consommateurs suisses sont de plus en plus conscients des liens entre commerce, développement et environnement, et ils veulent être informés sur l'origine et les conditions de production des biens provenant des pays en développement. Aussi la Suisse collabore-t-elle aux travaux multilatéraux visant une transparence aussi large que possible sur l'origine et les conditions de production de certains produits "éco-sensibles" (bois tropicaux p. ex.). Elle étudie ainsi l'introduction de labels facultatifs, par exemple pour l'importation de bois tropicaux produits dans des conditions de développement durable et pour d'autres produits.

Dans le cadre de mesures nationales en faveur de l'environnement et de limitations apportées au commerce, la Suisse veille à éviter des mesures ("protectionnisme écologique") qui peuvent avoir des effets négatifs sur l'économie des pays en développement et, à long terme, souvent aussi sur leur environnement. Les pays en développement qui, pour des raisons financières et techni- 
ques, ne sont pas encore en mesure d'atteindre des normes écologiques adéquates méritent un soutien pour améliorer leurs méthodes de production et de transformation.

\subsubsection{Aménager des politiques économique, commerciale et extérieure et des politiques de développement cohérentes sur le plan multilatéral}

La coopération au développement bilatérale suisse traditionnelle conservera dans les années à venir une place prépondérante. Toutefois, nous devons être conscients que la Suisse, avec une participation de moins de deux pour cent à l'aide internationale totale, ne peut jouer qu'un rôle mineur. Alors que les problèmes dépassent de plus en plus fréquemment les frontières nationales, il est d'autant plus nécessaire, pour les petits États comme la Suisse, d'accorder leurs moyens avec les autres pays donateurs et avec les pays récipiendaires. Les forums multilatéraux sont les lieux privilégiés de négociation des problèmes de cohérence. Un engagement accru de la Suisse dans les organisations internationales est inévitable. Les canaux multilatéraux doivent être davantage mis à contribution, en particulier là où lá Suisse jouit d'une certaine influence et a le droit de vote. L'aménagement cohórent des aspects pertinents pour le développement de la politique commerciale et économique, de la politique agricole, de la politique de l'emploi, de la politique d'asile et d'immigration, se heurtera en Suisse à moins de résistance si cet aménagement est concerté avec d'autres États dans un dialogue multilatéral. 